\title{
Prostate cancer cells produce exosomes modulating metastasis to the bones
}

\begin{abstract}
Prostate cancer metastasis to the bone is a frightening cause of death world-wide. Patients do not live a functional and active life before they eventually die. The major reason for prostate cancer metastasis is the use of poor bio markers such as Prostate-specific antigen (PSA) and histopathological grading to diagnose prostate cancer incorrectly, indicating that a patient who has a stage four prostate has a benign tumor. New and effective markers for prostate cancer are urgently needed. The goal of this review is to examine modulation of prostate cancer $(\mathrm{PCa})$ exosome interaction with prostate cancer micro-environment as a major support system to prevent incurable bone cancer. Conventional prostate cancer therapy is weakened by exchange of growth factors between cancer cells and it surroundings, leading to increase tumor growth and metastasis to other parts of the body. The surrounding of tumor includes prostate cancer exosomes, which can be release under suitable condition. Basically, the interactions between prostate cancer exosomes, growth factors and cell micro-environment support uncontrollable metastasis of prostate cancer to the bone. PCa exosome modification may be the solution to the increasing patient death caused by prostate cancer metastasis to the bone. PCa exosome is in the fore-front of research as a biomarker for early prostate cancer detection.
\end{abstract}

Volume 8 Issue 2 - 2017

\author{
Toluleke Oloruntobi Famuyiwa, James Kumi \\ Diaka, Joubin Jebelli \\ Department of Biological Sciences, Florida Atlantic University, \\ USA
}

Correspondence: Toluleke Oloruntobi Famuyiwa, Department of Biological Sciences, Florida Atlantic University,3200 College Avenue, Davie, USA, Tel I (954) 5I 2-3875,

Email tfamuyiwa2014@fau.edu

Received: February 08, 2017| Published: June 30, 2018

\section{Mini review}

In $2017,1,688,780$ new cancer cases and 600,920 cancer deaths are projected to occur in the United States. ${ }^{1}$, this corresponds to about 1,650 deaths per day. ${ }^{1}$. One common cause of this daily death is prostate cancer metastasis to bone., ${ }^{2,3}$ Bone metastases occur in most tumor types but are most prevalent in cancers of the breast, prostate, and lung. ${ }^{4}$ These bone lesions can cause deadly skeletal conditions, including spinal cord or nerve root dislocation, bone surgery, hypercalcemia, pathologic fractures, and extreme bone pain requiring immediate radiotherapy. ${ }^{5}$ all of which can significantly lower the patient's quality of life..$^{6-9}$ and may negatively affect patient survival. ${ }^{10,11}$ Current research focus onreduction of pain, prevention of abnormal skeletal conditions, and maintenance of quality of life to managing patients with metastatic bone cancer. ${ }^{12}$ However, these efforts have failed because diagnosis most of bone cancer lead to incurable bone cancer. ${ }^{8}$. This review examines modulation of prostate cancer $(\mathrm{PCa})$ exosome interaction with prostate cancer microenvironment as a major support system to prevent incurable bone cancer.

Treatment of metastatic prostate cancer has proven to be very challenging as a result of sustained activity of the androgen receptor, ${ }^{13-15}$ aided by prostate cancer exosome activities. Although first stage of PCa can be treated successfully, one in three of the cases of PCa progress to a more advance form, which often leads to patient death. ${ }^{16}$ or abnormal bone structure in $80 \%$ of the patients. ${ }^{17}$ Prostate tumors can be inactive or very aggressive, often metastasizing to the bone and other organs, thereby causing significant morbidity and mortality. ${ }^{18}$.Bone cancer present a 5 -year survival rate of just $30 \%$ against $100 \%$ for restricted disease. ${ }^{17}$

The major challenge that leads to prostate cancer metastasis to the tissues such as the bone, is the failure to detect prostate cancer at the early stage. A serious clinical challenge in prostate cancer is the inability of current diagnostic tests, including prostate-specific antigen (PSA) screening and histopathological grading, to distinguish between malignant and benign tumors. ${ }^{19} \mathrm{~A}$ timely diagnosis of $\mathrm{PCa}$ is helpful in reducing death from prostate cancer metastasis. ${ }^{20}$ A protein mainly secreted by prostate cells, prostate specific antigen (PSA), has been used as a blood-based biomarker for prostate cancer for more than 50years.

PSA is present in normal prostatic secretions and its levels are often elevated in prostate cancer patients. ${ }^{21}$. Serum PSA levels have been utilized as a prostate cancer biomarker for over 21years and PSA screening has transform the clinical management of the disease. ${ }^{22}$ However, PSA has intrinsic limitations, including lack of specificity, leading to over-diagnosis and over-treatment of prostate cancer. Even though PSA is a high-end tool, it lacks specificity and is therefore not considered an excellent biomarker. ${ }^{23}$ Thus, new and specific markers for prostate cancer are highly needed.

Consequently, concerted efforts are being made on searching for alternative prostate cancer biomarkers, particularly those that can predict the aggressiveness of the disease and drive better treatment decisions. PCa exosome is in the fore-front of research as a biomarker for prostate cancer detection. A good understanding of PCaexosome as a biomarker will eliminate unnecessary prostate biopsies, and support the urologist in recommending best treatment practices.

Exosomes are found naturally in blood, urine, cerebrospinal fluid, breast milk, saliva, ascetic fluid, and amniotic fluid in very high numbers. ${ }^{24 \_27}$ Exosomes are tiny membrane vesicles with a double layered lipid released by most living cells. ${ }^{28-30}$ The release of exosome is potentially impacted by the sorting of the cargo inside the exosome. The mechanisms underlying the sorting of cargo into the intraluminal vesicles (ILVs) have not been explained in detail. Both endosomal sorting complex required for transport (ESCRT)-dependent and independent signals have been suggested to determine the sorting of exosomes. ${ }^{31}$

There are other important factors that impact exosome release 
in cancer; accumulation of intracellular $\mathrm{Ca} 2+$ resulting in increased exosome secretion, ${ }^{32}$ oncogenes and tumor suppressors regulating exosome secretion(p53-regulated protein tumor suppressor-activated pathway 6 (TSAP6) induces exosome secretion under stressed conditions), ${ }^{33}{ }^{35}$ low micro environmental $\mathrm{pH}$ increasing exosome secretion and uptake by recipient cells. ${ }^{36}$

Furthermore, enzymes control the secretion of exosomes. Heparanase is an enzyme with elevated level in cancer. Overexpression of heparanase promotes exosome secretion. ${ }^{37}$ Interestingly, exosomes from normal mammary epithelial cells inhibit exosome secretion by breast cancer cells, implicating a feedback control to maintain dynamic equilibrium. ${ }^{38}$ Similarly, treating prostate cancer cells with exosome from normal prostate cells has potential therapeutic effect.

Conventional prostate cancer therapy is weaken by exchange of growth factors between cancer cells and it surroundings, leading to increase tumor growth and metastasis to other parts of the body. ${ }^{39}$ Over the last 30 years, considerable amount of research has done in order to understand the exosome mediated cell-cell communication mechanisms. ${ }^{40}$ These efforts have revealed various new facets of material transport across biological membranes and have verified, to a great extent, the role of exosomes in disease development. ${ }^{24,41}$

Metastatic and therapy resistant cancers sustain on robust biological interaction networks arising from gene-gene, gene-microRNA (miRNA), protein-protein, parallel signaling as well as intracellular, intercellular and distant cell interactions. ${ }^{42,43}$ Cancer-derived exosomes facilitate the cancer cells and their microenvironment to form a site that support tumor growth..$^{44}{ }^{46}$ Moreover, exosome concentration in the plasma of prostate cancer patients measured by nanoparticle tracking analysis (NTA), was more than that in the plasma of noncancerous individuals..$^{47}$ The spread of oncogenes by exosomes and microvesicles secreted by tumor cells have also been reported. ${ }^{48}$ If release of prostate cancer exosomes continues in this trend, there will be uncontrollable prostate cancermetastasis to the bone.

Another factor that determines the impact of exosome is absorption of exosomes by the surrounding cells. The uptake of exosomes occurs through a well-defined process and is controlled by transmembrane proteins. ${ }^{49}$. Studies suggest that the tetraspanin-integrin complex help in specificity, permitting the adhesion of exosomes to the right cells.$^{50,51}$ Furthermore, a pro- inflammatory environment may potentiate the expression of receptor molecules such as ICAM1(Intercellular Adhesion Molecule 1) on the membrane surface, with high tendency for exosomes attach to the target cells. ${ }^{52}$ Although the specific mechanism that drive exosomes absorption is unknown, the activity of the T-cell receptor/CD3 complex and the chemokine receptor CXCR4 on exosomes of $\mathrm{T}$-cells propose a juxtracrine pathway through receptor-ligand linkage. ${ }^{53}$

Alternatively, exosomes can merge with the cell membrane of target cells, which leads to a release of their content into the cytoplasm. ${ }^{52,54}$ Cellular absorption of exosomes can occur by phagocytosis in an actin-cytoskeleton and phosphatidylinositol 3-kinase -associated manner. ${ }^{52}$ Further studies are needed to understand how exosomes are directed to PCa cells and whether exosome protein results in organotropism associated with metastatic disease.

It is important to investigate the link between exosome protein and the metastatic potential of cancer after treatment, as indicate by unfolding findings. Exosomes facilitated transport of proteins between tumor cells can result into chemo resistance, which means high metastatic potential. Exosomes from docetaxel-resistant prostate cancer cell lines can confer chemo resistance to non-resistant prostate cancer cell lines through exosomes facilitated transport of drug transporter, MDR $-1 .{ }^{55}$ These observations represent a new possibility of how cancerous cells may modulate the immune system, becoming chemo resistant with the help of exosomes.

This chemo-resistant property may be impacted through biochemical change of connective tissue via exosome protein activities. Exosomes from prostate cancer cells lines contain TGF$\beta 1$ protein, which is transported to receiver cells in a functional form. A Study have showed that TGF- $\beta 1$ expressing exosomes can initiate the development of fibroblasts to myofibroblasts. ${ }^{56}$ Myofibroblasts release considerable matrix remodeling proteins within the tumor microenvironment and contribute to tumor angiogenesis. ${ }^{57}$ Cancer exosome-induced stimulation of fibroblasts could favor tumor angiogenesis. In addition, cancer cells transfer membrane-bound EGFR to endothelial cells via exosomes. ${ }^{56}$ This transfer triggers the autocrine VEGF/VEGFR-2 pathway in endothelial cells and further enhance tumor angiogenesis (development of new blood vessels to support the tumor). Cancer cells also stimulate immune cells to enhance tumor invasion, tumor angiogenesis and distribution. ${ }^{58}$

Exosomes derived from cancer cells activate the immune cells capable of weakening the immune systems' fight against cancer. Tumor-derived exosomes switch on myeloid-derived suppressor cells (MDSC). ${ }^{59}$ MDSCs cause immunosuppression in cancer by downregulating the T cell feedback. ${ }^{60}$ Tumor-derived exosomes from various tumor cell lines enhance interleukin-6 (IL-6) production in MDSCs, through the activation of the Toll-like receptor 2 via the membrane-linked heat shock protein. ${ }^{59}$ IL- 6 release triggered autocrine phosphorylation of Stat 3 inside MDSCs, which enhance their immunosuppressive effect on immune system.

Moreover, studies have revealed that tumor-derived exosomes express Fas ligand. ${ }^{61}$ Fas containing exosomes can impact immunosuppression via apoptosis of tumor-reactive $\mathrm{CD} 8+\mathrm{T}$ lymphocytes. ${ }^{62,63}$ The dead tumor-reactive CD8+ T lymphocytes cannot attack the cancer cells in this state. Likewise, cancer cells release exosomes that increase the level of regulatory T (Treg) cells. ${ }^{62,64}$ Tregs cause immunosuppression in the tumor microenvironment by hindering the function of anti-tumorigenic T cells. ${ }^{65}$ and antigen presenting dendritic cells. ${ }^{66-68}$

Additionally, given that exosome secretion is triggered by an intracellular increase of calcium $(\mathrm{Ca} 2+),{ }^{32}$ combination therapy involving intracellular calcium inhibition will be effective against cancer. Immunotherapeutic autologous dendritic cell-derived exosomes. ${ }^{69,70}$ and intracellular calcium inhibition drugs may be effective in preventing cancer metastasis to the bone.

Furthermore, exosomes produced by melanoma cells function as vesicles to transfer the receptor tyrosine kinase (RTK) from melanoma cells to the bone marrow-derived progenitor cells, thereby enhancing the metastatic process. ${ }^{71}$ Exosome protein concentration was highest in melanoma patients with the most advanced form of the disease; additionally, late-stage individuals harboring proteindeficient exosomes had a survival advantage compared to those with protein-rich exosomes. ${ }^{72}$

Regardless of the protein content of exosome, cells are known to deliver proteins between the intracellular organelles via membrane vesicles containing compatiblereceptors to ensure traffic specificity; the results accumulated over the last ten years have demonstrated that a heterogeneous group of vesicles are also released from the 
cell surface and used as intercellular signalosomes in information exchange, even over a long distance. ${ }^{73}$. This information exchange is facilitated by molecules such as protein, $\mathrm{RNAs},{ }^{74}$ breakdown products of signaling pathways, viruses. ${ }^{75}$ and, even recently, miRNAs. ${ }^{76}$ during transportation involving exosomes.RNAs behave in a unique way, different from other exosome cargo. ${ }^{77}$ Several miRNA expression profiles have been reported for prostate cancer, showing altered expression levels in prostate cancer tissue as compared to normal tissue. ${ }^{78-83}$ Several cellular processes like proliferation, differentiation, and apoptosis are shown to be regulated by miRNAs ${ }^{84}$ and miRNAs are found abnormally expressed in many types of cancer. ${ }^{79,81,85-87}$

Notably, miRNAs are currently being investigated as prognostic and diagnostic tools for prostate and other types of cancer ${ }^{88,89}$ Studies on blood-based exosome miRNAs as biomarkers for prostate cancer are emerging, however most of these are at initiation stage, vary in method, and lack characterization in the form in which the extracellular miRNAs are found. ${ }^{90}$ This makes exosome RNA a potential focus in studies related to prevention of prostate cancer metastasis to the bone.

Besides RNA's, intracellular calcium ion level can also be used to detect $\mathrm{PCa}$ condition, majorlybecause calcium ions $(\mathrm{Ca} 2+)$ act as second messenger to regulate gene transcription, cell proliferation, migration and death of $\mathrm{PCa}$ cells. ${ }^{91}$ The extracellular calciumsensing receptor (CaSR) plays a major role in the maintenance of a physiological serum ionized calcium $(\mathrm{Ca} 2+)$ concentration by regulating the circulating levels of parathyroid hormone. ${ }^{92}$ The heterodimeric G-protein coupled CaSR is the most important receptor on the cell surface for detecting extracellular calcium. In normal tissue, CaSR is responsible for the physiological regulation of calcium homeostasis in several organs such as kidney, breast, gastrointestinal tract, bones and parathyroid glands. ${ }^{93}$ The binding of calcium on CaSR activates a variety of signaling pathways that are important for PCa cell survival. Although CaSR may also have a tumor suppressing capacity in e.g. gastric and colon cancer, it has been demonstrated to be involved in bone metastasis of several tumor entities, such as renal cell carcinoma, prostate carcinoma and breast cancer. ${ }^{94}$

Furthermore, $\mathrm{Ca} 2+$ is a chemopreventive agent for colon cancer. ${ }^{95,96}$ It acts at the molecular level to inhibit or delay carcinogenesis in the colon, however, the mechanism is not fully understood. A study found that CaSR is expressed in human colon epithelium..$^{97,98}$ Compared with normal colon crypt epithelial cells, a loss of CaSR expression is observed in differentiated carcinomas, whereas little or no CaSR expression is found in undifferentiated and invasive carcinomas. ${ }^{98}$ Thus, the expression of CaSR may be linked with either abnormal differentiation or malignant progressionof $\mathrm{PCa}$, or even both.

\section{Conclusion}

This review highlights exosomes as a substitute biomarker for traditional diagnostic test such as prostate specific antigen (PSA) testing and digital rectal examination (DRE). PSA is not dependable as a PCa biomarker and levels can be high because of other disease conditions. ${ }^{99}$ Therefore, more effective bio-markers are needed for $\mathrm{PCa}$ prognosis, possibly preventing metastasis to the bone. $\mathrm{PCa}$ exosome modification may be the solution to the increasing patient death caused prostate cancer metastasis to the bone. This revision considers prostate cancer exosomes study as a compelling study for decreasing the metastasis of prostate cancer to the bones.

\section{Acknowledgements}

None.

\section{Conflicts of interests}

The authors declare that there are no conflicts of interest.

\section{Funding}

None.

\section{References}

1. RL Siegel, KD Miller, A Jemal. Cancer statistics. CA. Cancer J. Clin TOC. 2017;67(1):7-30.

2. GD Roodman. Mechanisms of Bone Metastasis. $N$ Engl $J$ Med 2017;350:1655-1664.

3. RE Coleman. Metastatic bone disease:clinical features, pathophysiology and treatment strategies. Cancer Treat Rev. 2001;27(3):165-176.

4. RE Coleman. Bisphosphonates: clinical experience. Oncologist. 2004;9(4):14-27.

5. RE Coleman. Clinical Features of Metastatic Bone Disease and Risk of Skeletal Morbidity. Clin Cancer Res. 2006;12(20 Pt 2):6243s-6249s.

6. CL Addison, N Bouqanim, J Hilton, et al. A phase II, multicentre trial evaluating the efficacy of de-escalated bisphosphonate therapy in metastatic breast cancer patients at low-risk of skeletal-related events. Breast Cancer Res Treat. 2014;144(3):615-624.

7. A Wardley, N Davidson, $\mathrm{P}$ Barrett-Lee, et al. Zoledronic acid significantly improves pain scores and quality of life in breast cancer patients with bone metastases:a randomised, crossover study of community vs hospital bisphosphonate administration. $\mathrm{Br} J$ Cancer 2005;92(10):1869-1876.

8. S Li, Y Peng, ED Weinhandi, et al. Estimated number of prevalent cases of metastatic bone disease in the US adult population. Clin Epidemiol. 2012:4:87-93.

9. KP Weinfurt, LD Castel, Y Li, et al. Health-related quality of life among patients with breast cancer receiving zoledronic acid or pamidronate disodium for metastatic bone lesions. Med Care. 2004;42(2):164-175.

10. MG Oefelein, V Ricchiuti, W Conrad, et al. Skeletal fractures negatively correlate with overall survival in men with prostate cancer. J Urol 2002;168(3):1005-1007.

11. F Saad, A Lipton, R Cook, et al. Pathologic fractures correlate with reduced survival in patients with malignant bone disease. Cancer.2007;110(8):1860-1867.

12. JR Berenson, L Rajdev, M Broder. Treatment strategies for skeletal complications of cancer. Cancer Biol Ther. 2006;5(9):1074-1077.

13. PC Albertsen, JA Hanley, J Fine. 20-Year Outcomes Following Conservative Management of Clinically Localized Prostate Cancer JAMA. 2005;293(17):2095-2101.

14. MH Tan, J Li, HE Xu, et al. Androgen receptor:structure, role in prostate cancer and drug discovery. Acta Pharmacol Sin. 2015;36:3-23.

15. J Ferlay, I Soerjomataram, R Dikshit, et al. Cancer incidence and mortality worldwide:Sources, methods and major patterns in GLOBOCAN 2012 Int $J$ Cancer. 2015;136(5):E359-E386.

16. C Festuccia, P Muzi, D Millimaggi, et al. Molecular aspects of gefitinib antiproliferative and pro-apoptotic effects in PTEN-positive and PTEN-negative prostate cancer cell lines. Endocr Relat Cancer. 2005;12(4):983-998.

17. MN Thobe, RJ Clark, RO Bainer, et al. From prostate to bone:Key players in prostate cancer bone metastasis. Cancers (Basel). 2011;3(1):478-493.

18. RD Loberg, CJ Logothetis, ET Keller, et al. Pathogenesis and treatment of prostate cancer bone metastases: targeting the lethal phenotype. J Clin Oncol. 2005;23(32):8232-8241. 
19. RJ Bryant, FC Hamdy. Screening for Prostate Cancer: An Update. Eur Urol. 2008;53(1):37-44.

20. WJ Catalona, DS Smith, TL Ratliff, et al. Measurement of ProstateSpecific Antigen in Serum as a Screening Test for Prostate Cancer. $N$ Engl J Med. 1991;324(17):1156-1161.

21. H Lilja, D Ulmert, AJ Vickers. Prostate-specific antigen and prostate cancer:prediction, detection and monitoring. Nat Rev Cancer. 2008;8(4):268-278.

22. VM Velonas, HH Woo, CG dos Remedios, et al. Current status of biomarkers for prostate cancer. Int J Mol Sci. 2013;14(6):11034-11060.

23. L Nogueira, R Corradi, JA Eastham. Other biomarkers for detecting prostate cancer. BJU Int. 2010;105(2):166-169.

24. AV Vlassov, S Magdaleno, R Setterquist, et al. Exosomes: Current knowledge of their composition, biological functions, and diagnostic and therapeutic potentials. Biochim Biophys Acta. 2012;1820(7):940 948.

25. L Balaj, R Lessard, L Dai, et al. Tumour microvesicles contain retrotransposon elements and amplified oncogene sequences. Nat Commun. 2011;2:180

26. C Théry, L Zitvogel, S Amigorena. Exosomes: composition, biogenesis and function. Nat Rev Immunol. 2002;2(8):569-579.

27. C Théry (2011) Exosomes:secreted vesicles and intercellular communications. F1000 Biol Reports. 2011;3:15.

28. S Keller, MP Sanderson, A Stoeck, et al. Exosomes: From biogenesis and secretion to biological function. Immunol Lett. 2006;107(2):102-108.

29. E Wieckowski, TL Whiteside. Human tumor-derived vs dendritic cellderived exosomes have distinct biologic roles and molecular profiles. Immunol Res. 2006;36(1-3):247-254.

30. G Camussi, MC Deregibus, S Bruno, et al. Exosomes/microvesicles as a mechanism of cell-to-cell communication. Kidney Int .2010;78(9):838848.

31. K Trajkovic, C Hsu, S Chiantia, et al. Ceramide Triggers Budding of Exosome Vesicles into Multivesicular Endosomes. Science. 2008;319(5867):1244-1247.

32. A Savina, M Furlán, M Vidal, et al. Exosome release is regulated by a calcium-dependent mechanism in K562 cells. J Biol Chem. 2003;278(22):20083-20090.

33. X Yu, SL Harris, AJ Levine. The regulation of exosome secretion: A novel function of the p53 protein. Cancer Res. 2006;66(9):4795-4801.

34. A Lespagnol, D Duflaut, C Beekman, et al. Exosome secretion, including the DNA damage-induced p53-dependent secretory pathway, is severely compromised in TSAP6/Steap3-null mice. Cell Death Differ. 2008;15(11):1723-1733.

35. JL Yu, May L, Lhotak V, et al. Oncogenic events regulate tissue factor expression in colorectal cancer cells:implications for tumor progression and angiogenesis. Blood. 2005;105(4):1734-1741.

36. I Parolini, Federici C, Raggi $\mathrm{C}$, et al. Microenvironmental $\mathrm{pH}$ is a key factor for exosome traffic in tumor cells. J Biol Chem. 2009;284(49):34211-34222.

37. CA Thompson, A Purushothaman, VC Ramani, et al. Heparanase regulates secretion, composition, and function of tumor cell-derived exosomes. J Biol Chem. 2013;288(14):10093-10099.

38. A Riches, E Campbell, E Borger, et al. Regulation of exosome release from mammary epithelial and breast cancer cells-A new regulatory pathway. Eur J Cancer. 2014;50(5):1025-1034.

39. R Kalluri, M Zeisberg (2006) Fibroblasts in cancer. Nat Rev Cancer. 2006;6(5):392-401.
40. RH Staals, GJ Pruijn. The human exosome and disease. Adv Exp Med Biol. 2011;702:132-142.

41. JS Schorey, S Bhatnagar. Exosome function:From tumor immunology to pathogen biology. Traffi. 2008;9(6):871-881

42. H Kitano.) Cancer as a robust system:implications for anticancer therapy. Nat Rev Cancer. 2004;4:227-235.

43. H Kitano. Cancer robustness:Tumour tactics. Nature .2003;426(6963):125.

44. HG Zhang, WE Grizzle. Exosomes:A novel pathway of local and distant intercellular communication that facilitates the growth and metastasis of neoplastic lesions. Am J Pathol. 2014;184(1):28-41.

45. DW Greening, Jingyi Sheng, Hong-Jian Zhu, et al. Emerging roles of exosomes during epithelial-mesenchymal transition and cancer progression. Semin Cell Dev Biol. 2015;40:60-71.

46. JL Hood, H Pan, GM Lanza, et al. Paracrine induction of endothelium by tumor exosomes. Lab Invest. 2009;89(11):1317-1328.

47. M Nawaz, Camussi G, Valadi $\mathrm{H}$, et al. The emerging role of extracellular vesicles as biomarkers for urogenital cancers. Nat Rev Urol. 2014;11(12):688-701.

48. K Al-Nedawi, Meehan B, Micallef J, et al. Intercellular transfer of the oncogenic receptor EGFRvIII by microvesicles derived from tumour cells. Nat Cell Biol. 2008;10(5):619-624.

49. D Feng, Zhao WL, Ye YY, et al. Cellular internalization of exosomes occurs through phagocytosis. Traffic. 2010;11(5):675-687.

50. I Nazarenko, Rana S, Baumann A, et al. Cell surface tetraspanin Tspan8 contributes to molecular pathways of exosome-induced endothelial cell activation. Cancer Res. 2010;70(4):1668-1678.

51. S Rana, S Yue, D Stadel, et al. Toward tailored exosomes:The exosomal tetraspanin web contributes to target cell selection. Int J Biochem Cell Biol. 2012;44(9):1574-1584.

52. A Clayton. Adhesion and signaling by B cell-derived exosomes:the role of integrins. FASEB J. 2004;18(9):977-979.

53. N Blanchard, Lankar D, Faure F, et al. TCR Activation of Human T Cells Induces the Production of Exosomes Bearing the TCR/CD3/zeta Complex. J Immunol. 2002;168(7):3235-3241.

54. A Montecalvo, Larregina AT, Shufesky WJ, et al. Mechanism of transfer of functional microRNAs between mouse dendritic cells via exosomes. Blood. 2012;119(3):756-766.

55. C Corcoran, Rani S, O’Brien K, et al. Docetaxel-Resistance in Prostate Cancer:Evaluating Associated Phenotypic Changes and Potential for Resistance Transfer via Exosomes. PLoS One. 2012;7(12):e50999.

56. J Webber, R Steadman, MD Mason, et al. Cancer exosomes trigger fibroblast to myofibroblast differentiation. Cancer Res. 2010;70(23):9621-9630.

57. S Vong, R Kalluri (2011) The Role of Stromal Myofibroblast and Extracellular Matrix in Tumor Angiogenesis. Genes Cancer. 2011;2(12):1139-1145.

58. JA Joyce, JW Pollard. Microenvironmental regulation of metastasis. Nat Rev Cancer. 2009;9(4):239-252.

59. F Chalmin, Ladoire S, Mignot $G$, et al. Membrane-associated Hsp72 from tumor-derived exosomes mediates STAT3-dependent immunosuppressive function of mouse and human myeloid-derived suppressor cells. J Clin Invest. 2010;120(2):457-471.

60. S Nagaraj, DI Gabrilovich. Regulation of suppressive function of myeloid-derived suppressor cells by CD4+ T cells. Semin Cancer Biol. 2012;22(4):282-288. 
61. MJ Martínez-Lorenzo, Anel A, Alava MA, et al. The human melanoma cell line MelJuSo secretes bioactive FasL and APO2L/TRAIL on the surface of microvesicles. Possible contribution to tumor counterattack. Exp Cell Res. 2004;295(2):315-329.

62. EU Wieckowski, Visus C, Szajnik M, et al. Tumor-Derived Microvesicles Promote Regulatory $\mathrm{T}$ Cell Expansion and Induce Apoptosis in Tumor-Reactive Activated CD8+ T Lymphocytes. $J$ Immunol. 2009;183(6):3720-3730.

63. AJ Abusamra, Zhong Z, Zheng X, et al. Tumor exosomes expressing Fas ligand mediate CD8+ T-cell apoptosis. Blood Cells Mol Dis. 2005;35(2):169-173.

64. M Szajnik, M Czystowska, MJ Szczepanski, et al. Tumor-derived microvesicles induce, expand and up-regulate biological activities of human regulatory T cells (Treg). PLoS One .2010;5(7):e11469.

65. D Lindau, P Gielen, M Kroesen, et al. The immunosuppressive tumour network:myeloid-derived suppressor cells, regulatory $\mathrm{T}$ cells and natural killer T cells. Immunology. 2012;138(2):105-115.

66. F Andre, Schartz NE, Movassagh M, et al. Malignant effusions and immunogenic tumour-derived exosomes. Lancet. 2002;360(9329):295305 .

67. F André, Schartz NE, Chaput N, et al. Tumor-derived exosomes:a new source of tumor rejection antigens. Vaccine. 2002;20(4):A28-31.

68. DD Taylor, C Gercel-Taylor. MicroRNA signatures of tumor-derived exosomes as diagnostic biomarkers of ovarian cancer. Gynecol Oncol. 2008;110(1):13-21.

69. F Belli, Testori A, Rivoltini L, et al. Vaccination of metastatic melanoma patients with autologous tumor-derived heat shock protein gp96peptide complexes:Clinical and immunologic findings. J Clin Oncol. 2002;20(20):4169-4180.

70. MA Morse, Garst J, Osada T, et al. A phase I study of dexosome immunotherapy in patients with advanced non-small cell lung cancer. $J$ Transl Med. 2005;3(1):9.

71. Y Qin, R Sun, C Wu, et al. Exosome:A novel approach to stimulate bone regeneration through regulation of osteogenesis and angiogenesis. Int $J$ Mol Sci. 2002;17(5):E712.

72. H Peinado, S Lavotshkin, D Lyden. The secreted factors responsible for pre-metastatic niche formation:Old sayings and new thoughts. Semin Cancer Biol. 2011;21(2):139-146.

73. M Simons, G Raposo. Exosomes - vesicular carriers for intercellular communication. Curr Opin Cell Biol. 2009;21(4):575-581.

74. P Vinciguerra, F Stutz. mRNA export:An assembly line from genes to nuclear pores. Curr Opin Cell Biol. 2004;16(3):285-292.

75. B Février, D Vilette, H Laude, G Raposo. Exosomes:A bubble ride for prions? Traffic. 2005;6(1):10-17.

76. DJ Gibbings, C Ciaudo, M Erhardt, et al. Multivesicular bodies associate with components of miRNA effector complexes and modulate miRNA activity. Nat Cell Biol. 2009;11(9):1143-1149.

77. H Valadi, Ekström K, Bossios A, et al. Exosome-mediated transfer of mRNAs and microRNAs is a novel mechanism of genetic exchange between cells. Nat Cell Biol. 2007;9(6):654-659.

78. MJ Lodes, Caraballo M, Suciu D, et al. Detection of cancer with serum miRNAs on an oligonucleotide microarray. PLoS One. 2009;4(7):e6229.

79. S Volinia, Calin GA, Liu CG, et al. A microRNA expression signature of human solid tumors defines cancer gene targets. Proc Natl Acad Sci U S A. 2006;103(7):2257-2261
80. M Ozen, CJ Creighton, M Ozdemir, et al. Widespread deregulation of microRNA expression in human prostate cancer. Oncogene. 2008;27(12):1788-1793.

81. KP Porkka, Minja J Pfeiffer, Kati K Waltering, et al. MicroRNA expression profiling in prostate cancer. Cancer Res. 2007;67(13):6130 6135.

82. S Ambs, Prueitt RL, Yi M, et al. Genomic profiling of microRNA and messenger RNA reveals deregulated microRNA expression in prostate cancer. Cancer Res. 2008;68(15):6162-6170

83. J Szczyrba, Löprich E, Wach S, et al. (The MicroRNA Profile of Prostate Carcinoma Obtained by Deep Sequencing. Mol Cancer Res. 2010;8(4):529-538.

84. DP Bartel. MicroRNAs:Genomics, Biogenesis, Mechanism, and Function. Cell. 2004;116(2):281-297.

85. GA Calin, Ferracin M, Cimmino A, et al. A MicroRNA signature associated with prognosis and progression in chronic lymphocytic leukemia. N Engl J Med. 2005;353(17):1793-1801.

86. J Lu, Getz G, Miska EA, et al. MicroRNA expression profiles classify human cancers. Nature. 2005;435(7043):834-838.

87. H Suzuki, R Maruyama, E Yamamoto, et al. DNA methylation and microRNA dysregulation in cancer. Mol Oncol. 2012;6(6):567-578

88. KD Sørensen, TF Ørntoft. Discovery of prostate cancer biomarkers by microarray gene expression profiling. Expert Rev Mol Diagn. 2010;10(1):49-64.

89. R Kuner, JC Brase, H Sültmann, et al. MicroRNA biomarkers in body fluids of prostate cancer patients. Methods. 2013;59(1):132-137.

90. NP Hessvik, K Sandvig, A Llorente (2013) Exosomal miRNAs as biomarkers for prostate cancer. Front Genet. 2013;4:36.

91. C Cui, R Merritt, L Fu, Z Pan (2017) Targeting calcium signaling in cancer therapy. Acta Pharm Sin B. 2017;7(1):3-17.

92. D Riccardi, EM Brown (2010) Physiology and pathophysiology of the calcium-sensing receptor in the kidney. AJP Ren Physiol. 2010;298(3):F485-F499.

93. S Tennakoon, A Aggarwal, E Kállay. The calcium-sensing receptor and the hallmarks of cancer. Biochim Biophys Acta. 2015;1863(6):13981407.

94. CH Tae, Shim KN, Kim HI, et al. Significance of calcium-sensing receptor expression in gastric cancer. Scand $J$ Gastroenterol. 2016;51(1):67-72

95. M Lipkin. Preclinical and early human studies of calcium and colon cancer prevention. Ann N Y Acad Sci. 1999;889:120-127.

96. MJ Wargovich, Jimenez A, McKee K, et al. Efficacy of potential chemopreventive agents on rat colon aberrant crypt formation and progression. Carcinogenesis. 2000;21(6):1149-1155.

97. Y Sheinin, Enikö Kállay, Friedrich Wrba, et al. Immunocytochemical localization of the extracellular calcium-sensing receptor in normal and malignant human large intestinal mucosa. $J$ Histochem Cytochem. 2000;48(5):595-601.

98. S Chakrabarty, V Radjendirane, H Appelman, et al. Extracellular Calcium and Calcium Sensing Receptor Function in Human Colon Carcinomas:Promotion of E-Cadherin Expression and Suppression of beta-Catenin/TCF Activation. CANCER Res. 2003;63(1):67-71.

99. F Tarhan, A Orçun, I Küçükercan, et al. Effect of prostatic massage on serum complexed prostate-specific antigen levels. Urology. 2005;66(6):1234-1238. 